\title{
Estudo comparativo da resistência à fratura de pré-molares superiores íntegros e restaurados com amálgama aderido ${ }^{\dagger}$
}

\section{Comparative study of the fracture resistance of sound upper premolars and upper premolars restored with bonded amalgam}

\author{
André Marcelo Peruchi Minto* \\ Welingtom Dinelli** \\ Tomio Nonaka*** \\ Luis Henrique de Camargo Thomé****
}

\begin{abstract}
RESUMO: No presente estudo avaliou-se, in vitro, a resistência à fratura de pré-molares superiores preparados com cavidades de classe II compostas (conservadoras e extensas) restauradas com amálgama aderido a dois tipos diferentes de sistemas adesivos. Setenta dentes foram divididos em 4 grupos: grupo 1 ou controle com 10 dentes integros; grupo 2 com 20 dentes, sendo 10 cavidades conservadoras e 10 extensas restauradas com amálgama sem qualquer tipo de forramento. O grupo 3 e o grupo 4 foram compostos da mesma forma que o grupo 2, sendo que o primeiro recebeu cimento de ionômero de vidro (Vitrebond - 3M) e o segundo, adesivo dental (Scotchbond Multi-Purpose Plus - 3M), antes de serem restaurados. Os dentes haviam sido incluídos anteriormente em cilindros de PVC e fixados com resina acrílica. Após serem restaurados e termociclados, foram submetidos à fratura por força de compressão em uma máquina universal de testes EMIC-MEM 2000. Após análise de variância e aplicação do teste complementar de Tukey, concluiu-se que os sistemas adesivos utilizados condicionaram o aumento da resistência à fratura da estrutura dental nas cavidades convencionais, sendo os dentes com cavidades conservadoras mais resistentes em qualquer condição experimental.
\end{abstract}

UNITERMOS: Resistência à fratura; Preparo da cavidade dentária; Amálgama dentário; Adesivos dentinários; Bicúspide.

\begin{abstract}
The purpose of this in vitro study was to determine the fracture resistance of upper premolars which had received class II preparations (conservative and extensive) and were restored with bonded amalgam, with two different adhesive systems. Seventy teeth were divided in four groups: group 1 (control), with ten sound teeth; group 2, with twenty prepared teeth (10 teeth received conservative cavities and 10, extensive cavities) restored with amalgam without any kind of liner; groups 3 and 4, similar to group 2, though with linings of glass ionomer cement (Vitrebond 3M) (group 3) and dental adhesive (Scotchbond Multi-Purpose Plus - 3M) (group 4). The teeth were previously fixed in PVC cylinders with acrylic resin. After being restored and thermocycled, the test specimens were submitted to fracture by means of compression in an EMIC-MEM 2000 universal testing machine. After the application of the analysis of variance and complementary Tukey's test, we concluded that the utilized adhesive systems produced an increase of the fracture resistance of teeth presenting with conventional cavities; the teeth presenting with conservative cavities were more resistant in all experimental situations.
\end{abstract}

UNITERMS: Resistance to fracture; Dental cavity preparation; Dental amalgam; Dentin-bonding agents; Bicuspid.

\section{INTRODUÇÃO}

O amálgama do sistema prata-estanho é tradicionalmente aceito como material restaurador, sendo sua longevidade inigualável entre os materiais de aplicação direta.

De acordo com os principios fundamentais do preparo cavitário estabelecidos por Black ${ }^{2}$ (1920), as cavidades para amálgama deveriam ter largura ideal de $1 / 3$ da distância entre as cúspides com paredes circundantes vestibular e lingual paralelas entre si. Black ${ }^{2}$ (1920) também estabeleceu que o amálgama deveria permanecer nas cavidades por meio de retenções mecânicas e, à luz do conhecimento e das técnicas utilizadas na época, instituiu o conceito de "extensão preventiva", preocu-

\footnotetext{
$\dagger$ Resumo de Dissertação de Mestrado.

*Professor Adjunto da Disciplina de Dentística Restauradora da Faculdade de Odontologia da Universidade de Ribeirão Preto.

**Professor Titular da Faculdade de Odontologia de Araraquara da Universidade Estadual Paulista "Júlio de Mesquita Filho".

*** Professor Assistente Doutor; ${ }^{* * * *}$ Professor Assistente - Faculdade de Odontologia de Ribeirão Preto da Universidade de São Paulo.
} 
Minto AMP, Dinelli W, Nonaka T, Thomé LH de C. Estudo comparativo da resistência à fratura de pré-molares superiores íntegros e restaurados com amálgama aderido. Pesqui Odontol Bras 2002;16(2):121-126.

pado com a reincidência de cárie nas margens da restauração, conceito este que demandava desgaste excessivo da estrutura dental sadia.

Em virtude da redução da resistência da estrutura dental que ocorre frente aos preparos convencionais, Markley ${ }^{19}$ (1951) modificou drasticamente os conceitos de Black ${ }^{2}$ (1920), adotando preparos mais conservadores. Os trabalhos de Markley ${ }^{19}$ (1951) ganharam notoriedade, uma vez que somente em 1959, Vale $^{29}$ estabeleceu a relação entre os tipos de preparações cavitárias e a conseqüente redução da resistência da estrutura dental.

Com o advento da técnica de condicionamento ácido desenvolvida por Buonocore ${ }^{5}$ (1955), inicia-se a fase da Odontologia Adesiva. Desse modo, Varga et al..$^{30}$, em 1986, demonstraram que o amálgama pode ser retido na cavidade por meio de um sistema resinoso cujos componentes aderem-se ao esmalte. Além disso, demonstraram que a microinfiltração marginal pode ser reduzida pelo uso de adesivos resinosos.

Dentro da nova técnica que se apresentava, seguiram-se três requisitos fundamentais para conduzir ao sucesso da restauração: 1) reduzir a microinfiltração marginal ${ }^{26,27,28}$; 2) ser capaz de promover retenção auxiliar ${ }^{8,18,26}$; 3) elevar a resistência da estrutura dental enfraquecida pelo preparo cavitário9.

Dentro deste contexto, é propósito deste estudo comparar in vitro a resistência à fratura de dentes pré-molares superiores com cavidades de classe II compostas.

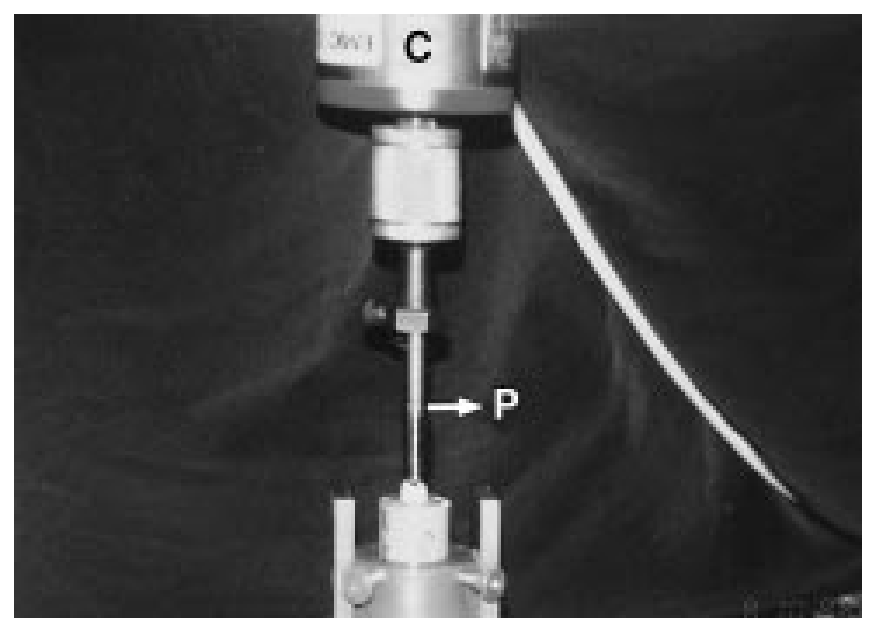

FIGURA 1 - Corpo-de-prova posicionado para teste de ensaio mecânico: P) pino multifuncional; C) célula de carga.

\section{MATERIAL E MÉTODO}

De um banco de dentes, foram selecionados 70 pré-molares superiores hígidos com dimensões semelhantes, extraídos por razões ortodônticas. Para a avaliação da higidez, foi utilizada uma lupa esterioscópica e as dimensões V-L $(9,0 \mathrm{~mm})$ e M-D $(7,0 \mathrm{~mm})$ foram padronizadas por meio de um paquímetro digital, podendo haver variações de até 1,0 mm em ambas dimensões. Após a limpeza dos dentes com instrumentos periodontais manuais e ultrassônico, estes foram incluídos em cilindros de PVC, preenchidos com resina acrílica até $1,0 \mathrm{~mm}$ aquém do colo dental (Figura 1). Os dentes agora montados foram divididos em 4 grupos experimentais da seguinte maneira:

- Grupo 1: 10 dentes hígidos que não receberam qualquer tipo de preparo considerados como pertencentes ao grupo controle.

- Grupo 2: 20 dentes com cavidades de classe II compostas, sendo 10 cavidades conservadoras e 10 cavidades convencionais. Todas as cavidades foram restauradas com amálgama de partículas esféricas com alto teor em cobre*, sem nenhum tratamento prévio.

- Grupo 3: 20 dentes com cavidades de classe II compostas, sendo 10 cavidades conservadoras e 10 cavidades convencionais. Todas as cavidades foram restauradas com amálgama, pela técnica adesiva com cimento de ionômero de vi-

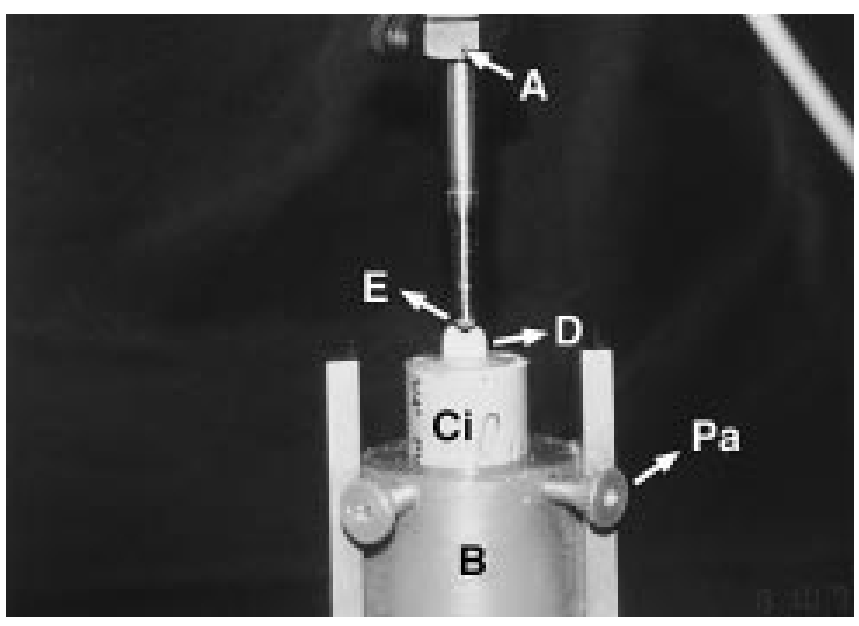

FIGURA 2 - Corpo-de-prova posicionado para teste de ensaio mecânico: A) adaptador para o pino multifuncional; B) base de latão para fixar o cilindro; $\mathrm{Pa}$ ) parafuso de fixação do cilindro localizado na base de latão; Ci) cilindro de PVC; D) dente utilizado como corpo-de-prova; E) esfera de aço com $5 \mathrm{~mm}$ de diâmetro afixada à ponta do pino.

*GS-80/SDI-Austrália. 
Minto AMP, Dinelli W, Nonaka T, Thomé LH de C. Estudo comparativo da resistência à fratura de pré-molares superiores íntegros e restaurados com amálgama aderido. Pesqui Odontol Bras 2002;16(2):121-126.

dro*. A cavidade era pré-tratada com ácido poliacrílico**, o cimento de ionômero era aplicado com um pincel em fina camada e o amálgama condensado antes que o cimento pudesse tomar presa.

- Grupo 4: 20 dentes com cavidades de classe II compostas, sendo 10 cavidades conservadoras e 10 cavidades convencionais. Todas as cavidades foram restauradas com amálgama, pela técnica adesiva, utilizando-se adesivo dental sem carga inorgânica***, seguindo-se as instruções do fabricante.

É importante salientar que as cavidades foram padronizadas tanto em largura quanto em profundidade em cada uma de suas caixas, por meio da utilização do dispositivo preconizado por Sá; Gabrielli $^{25}$ (1979). As cavidades convencionais possuíam caixas oclusais com $3,0 \mathrm{~mm}$ de largura $\mathrm{x}$ $3,0 \mathrm{~mm}$ de profundidade, e caixas proximais com $3,0 \mathrm{~mm}$ de largura $\times 2,5 \mathrm{~mm}$ de profundidade $\mathrm{x}$ $5,0 \mathrm{~mm}$ de altura. Todas as cavidades conservadoras tiveram suas dimensões reduzidas em 1,0 mm em relação às convencionais, sendo que para a realização dos preparos foi utilizada uma fresa "carbide" de alta rotação $\mathrm{n}^{\circ} 245$, sob refrigeração abundante.

O amálgama foi condensado com condensadores manuais, utilizando-se um porta matriz tipo Tofflemire. Após a condensação, o amálgama foi brunido e a restauração esculpida com uma espátula de Hollenback 3S.

Terminadas todas as restaurações, os corpos-de-prova foram termociclados por banhos de trinta segundos em água, com temperatura variando de $5^{\circ}$ a $55^{\circ} \mathrm{C}$, perfazendo-se um total de 250 ciclos. Após a termociclagem, os corpos-de-prova foram acondicionados em um recipiente com água destilada à temperatura ambiente por $48 \mathrm{~h}$. Decorrido este periodo, cada um dos corpos-de-prova foi fixado à uma máquina universal de ensaios mecânicos ${ }^{* * * *}$ e submetido a carga de compressão por meio de uma esfera de 5,0 $\mathrm{mm}$ de diâmetro presa a um pino de aço. A esfera tocava somente os planos inclinados das cúspides em estrutura dental e a pressão era realizada a uma velocidade de $0,5 \mathrm{~mm} / \mathrm{min}$, constante, até que ocorresse a fratura do corpo-de-prova (Figuras 1 e 2). Os resultados das cargas necessárias para que ocorresse a fratu- ra eram registrados pela máquina em kgf e anotados em fichas apropriadas.

\section{RESULTADOS}

Os resultados originais obtidos após os testes mecânicos foram submetidos a análise de variância para estabelecer a presença de diferenças estatísticas, entre os grupos (Tabela 1).

Utilizando-se o método de Tukey, em que o nível de significância adotado foi de $5 \%$, procedeu-se à comparação das médias entre os grupos (Tabela 2).

Pela análise do Gráfico 1, pode-se observar a elevação da resistência da estrutura dental nas cavidades convencionais quando se usa a técnica do amálgama aderido. Nota-se também que as cavidades conservadoras não apresentaram diferenças significantes em relação ao grupo controle, em qualquer condição experimental.

Os resultados obtidos referem-se aos testes efetuados em 70 corpos-de-prova, sendo 10 réplicas para cada condição experimental, onde: $\mathrm{C}=$ grupo controle; $\mathrm{ESF}$ = cavidades extensas sem forramento; EVITRE = cavidades extensas com Vitrebond; ESBMP = cavidades extensas com Scotchbond Multi-Purpose Plus; CSF = cavidades conservadoras sem forramento; CVITRE = cavidades conservadoras com Vitrebond; CSBMP = cavidades conservadoras com Scotchbond Multi-Purpose Plus.

\section{DISCUSSÃO}

Varga et $a .^{30}$, em 1986, descreveu a técnica de realização de restaurações de amálgama juntamente com o adesivo dental. A partir daí, as vantagens desse procedimento vêm sendo relatadas com ênfase pela literatura, demonstrando a capacidade do amálgama aderido em reduzir a microinfiltração marginal, promover retenção auxiliar e elevar a resistência da estrutura dental, à medida que novos materiais vão sendo introduzidos no mercado.

TABELA 1 - Análise de variância.

\begin{tabular}{l|c|c|c|c}
\hline \hline Fonte de variação & GL & SQ & QM & F \\
\hline Entre os grupos & 6 & $27.275,8510$ & $4.545,9752$ & $30,4237^{*}$ \\
\hline Dentro dos grupos & 63 & $9.413,5881$ & 149,4220 & \\
\hline Total & 69 & $36.689,4391$ & & \\
\hline \hline
\end{tabular}

*Significativo.

\footnotetext{
*Vitrebond - 3M.

**Condicionador de Dentina - SS White.

*** Scotchbond Multi-Purpose Plus - 3M.

****EMIC-MEM 2000.
} 
Minto AMP, Dinelli W, Nonaka T, Thomé LH de C. Estudo comparativo da resistência à fratura de pré-molares superiores íntegros e restaurados com amálgama aderido. Pesqui Odontol Bras 2002;16(2):121-126.

TABELA 2 - Contrastes estatísticos entre os grupos.

\begin{tabular}{|c|c|c|}
\hline Contraste & $\begin{array}{l}\text { Diferença entre } \\
\text { as médias }\end{array}$ & $\begin{array}{c}\text { Valor crítico de } \\
\text { Tukey }\end{array}$ \\
\hline $\mathrm{C} \times \mathrm{ESF}$ & $47,13^{*}$ & 16,68 \\
\hline C x EVITRE & $40,62 *$ & \\
\hline $\mathrm{C} \times \mathrm{ESBMP}$ & $24,61 *$ & \\
\hline $\mathrm{C} \times \mathrm{CSF}$ & 7,46 & \\
\hline C x CVITRE & 0,02 & \\
\hline $\mathrm{C} \times \mathrm{CSBMP}$ & 6,20 & \\
\hline ESF $x$ EVITRE & 6,51 & \\
\hline ESF x ESBMP & $22,52 *$ & \\
\hline ESF $x$ CSF & $39,67 *$ & \\
\hline ESF x CVITRE & $47,15^{*}$ & \\
\hline ESF x CSBMP & $53,33^{*}$ & \\
\hline EVITRE $x$ ESBMP & 16,01 & \\
\hline EVITRE x CSF & $33,26^{*}$ & \\
\hline EVITRE $x$ CVITRE & $40,64 *$ & \\
\hline EVITRE x CSBMP & $46,82 *$ & \\
\hline ESBMP x CSF & $17,15^{*}$ & \\
\hline ESBMP x CVITRE & $24,63 *$ & \\
\hline ESBMP x CSBMP & $30,81 *$ & \\
\hline CSF x CVITRE & 7,48 & \\
\hline CSF x CSBMP & 13,66 & \\
\hline CVITRE x CSBMP & 6,18 & \\
\hline
\end{tabular}

*Diferença significativa. $\mathrm{C}=$ grupo controle; $\mathrm{ESF}=$ cavidades extensas sem forramento; EVITRE = cavidades extensas com Vitrebond; ESBMP = cavidades extensas com Scotchbond Multi-Purpose Plus; CSF = cavidades conservadoras sem forramento; CVITRE = cavidades conservadoras com Vitrebond; CSBMP = cavidades conservadoras com Scotchbond Multi-Purpose Plus.

Os maiores problemas relacionados às restaurações de amálgama, segundo Eakle $^{9}$ (1986), são as fraturas marginais e as fraturas dentais, que podem resultar em aumento da microinfiltração e numa possivel recidiva de cárie. De acordo com Hasegawa; Retief ${ }^{14}$ (1996), esses problemas são desencadeados, em parte, pela falta de adesão do amálgama às paredes cavitárias. Assim sendo, Eakle $^{10}$, em 1992, demonstrou que a utilização do cimento resinoso Panavia como agente adesivo aumentava a força necessária para fraturar a estrutura dental contendo cavidades clássicas tipo MOD, quando comparadas com o grupo sem ade-

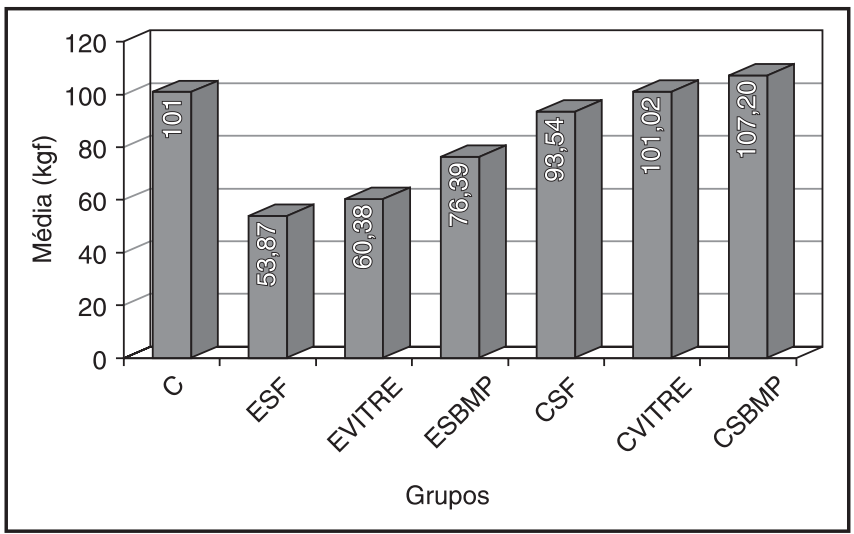

GRÁFICO 1 - Média da resistência à compressão da estrutura dental em função do tratamento da cavidade. $\mathrm{C}=$ grupo controle; $\mathrm{ESF}$ = cavidades extensas sem forramento; EVITRE = cavidades extensas com Vitrebond; ESBMP = cavidades extensas com Scotchbond Multi-Purpose Plus; CSF = cavidades conservadoras sem forramento; CVITRE = cavidades conservadoras com Vitrebond; CSBMP = cavidades conservadoras com Scotchbond Multi-Purpose Plus.

sivo. Este trabalho foi referendado por outros autores como Staninec; Holt ${ }^{27}$ (1988), que mostraram a união efetiva que havia entre o amálgama e o cimento Panavia, advindo daí alguns beneficios, tais como inibição da microinfiltração marginal inicial e diminuição do tamanho do preparo cavitário. Outras possibilidades ainda seriam abordadas, tais como proporcionar suporte às cúspides enfraquecidas, melhorar a resistência à fratura de modo a permitir a restauração de dentes comprometidos estruturalmente.

Essa última hipótese levantada por Staninec; Holt $^{27}$ (1988) vem ao encontro dos resultados obtidos por este estudo, visto que pela análise do Gráfico 1 , nota-se que o efeito de reforço da estrutura dental proporcionado pelos sistemas adesivos faz-se mais evidente em cavidades convencionais, onde há maior perda de estrutura dental. No presente trabalho, optou-se por empregar dois materiais como sistemas adesivos, os quais são mais comumente usados na técnica de amálgama aderido: o cimento de ionômero de vidro (Vitrebond $3 \mathrm{M})$ e o adesivo dentinário "dual" (Scotchbond Multi-Purpose Plus - 3M).

Dentro desse contexto o adesivo dentinário utilizado destacou-se como o material que propiciou maior aumento da resistência da estrutura dental, em concordância com outros autores ${ }^{3,4,11,13,15,16}$.

Quando se compararam as cavidades conservadoras com e sem o uso de sistemas adesivos, sejam eles cimento de ionômero de vidro ou adesivo den- 
Minto AMP, Dinelli W, Nonaka T, Thomé LH de C. Estudo comparativo da resistência à fratura de pré-molares superiores íntegros e restaurados com amálgama aderido. Pesqui Odontol Bras 2002;16(2):121-126.

tinário, não foram observadas diferenças estatisticamente significantes na elevação da resistência, demonstrando ainda a importância da limitação da forma de contorno nos preparos para amálgama. Nesse ponto, os resultados são corroborados pelo pioneiro trabalho de $\operatorname{Vale}^{29}(1959)$ e posteriormente pelos trabalhos de Mondelli21 ${ }^{21}$ (1980), ElMowafy $^{12}$ (1993) e Caron ${ }^{7}$ (1996).

Os testes de adesividade são freqüentemente realizados quando se busca obter forças de adesão ao esmalte e à dentina cada vez maiores.

Esta idéia justifica a utilização de um adesivo capaz de formar a camada híbrida, descrita por Nakabayashi et al. ${ }^{22}$ (1991), obtendo-se assim efetiva impermeabilização da dentina e máxima retenção do agente adesivo à estrutura dental.

Ramos; Perdigão ${ }^{23}$, em 1997, salientaram a necessidade de se condensar o amálgama antes que o adesivo viesse a se polimerizar para que houvesse a evidente união micromecânica entre os materiais. Isso realça a necessidade da utilização de um adesivo de polimerização química ou "dual", como o Allbond $2^{1,17,20,24}$ e o Scotchbond Multi-Purpose Plus $s^{6,20,24,31}$. O fato é que, uma vez unido às paredes cavitárias, mesmo que com mínima força de adesão, a carga sofrida pela restauração é distribuída por todas as paredes do preparo no sentido de unir as cúspides e não de afastá-las.

De modo geral, e em função das informações contidas na literatura, salientam-se várias vantagens da técnica de amálgama aderido, resultantes

\section{REFERÊNCIAS}

1. Bailey R, Boyer D. Influence of bonding on fracture resistance of class I amalgam restorations. J Dent Res 1997; $76: 67$.

2. Black GV. Oper Dent 1920;2:128-33.

3. Boyer DB, Roth L. Fracture resistance of teeth with bonded amalgams. Am J Dent 1994;7:91-4.

4. Borchert E, Boyer DB. Reinforcement of molars of bonded MOD amalgam restorations [abstract 1262] J Dent Res 1996;75:175.

5. Buonocore MG. A simple method of increasing the adhesion of acrylic filling materials to enamel surfaces. J Dent Res 1955;34;849-53.

6. Burgess JO, Alvarez AN, Summitt JB. Fracture resistance of complex amalgam. J Dent Res 1993;72:132.

7. Caron GA. Resistance to fracture of teeth with various preparations for amalgam. J Dent 1996;24:407-10.

8. Charlton DG, Moore BK, Swartz ML. In vitro evaluation of the use of resin liners to reduce microleakage and improve retention of amalgam restorations. Oper Dent 1992; 17:112-9.

9. Eakle WS. Fracture resistance of teeth restored with class II bonded composite resin. J Dent Res 1986;65:149-53. da união significativa da restauração à estrutura dental: 1) melhor retenção das restaurações; 2) reforço da estrutura dental; 3) menor microinfiltração marginal; 4) menor sensibilidade pós-operatória; 5) menor indice de fraturas marginais; 6) menor pigmentação da estrutura dental por ions metálicos; 7) menor corrosão.

\section{CONCLUSÕES}

Tendo em vista a análise dos resultados sob as condições experimentais apresentadas, é lícito concluir que:

1. quando comparadas com o grupo controle, as cavidades conservadoras condicionaram resistência à fratura da estrutura dental significativamente maior do que as cavidades convencionais;

2. as cavidades convencionais restauradas com amálgama sem o tratamento adesivo das paredes de esmalte-dentina, apresentavam severa redução da resistência em relação ao dente íntegro;

3. os melhores resultados de aumento da resistência à fratura foram obtidos nas cavidades convencionais restauradas com amálgama associado ao adesivo dental, seguida pela associação com o cimento de ionômero de vidro;

4. sob o aspecto de aumento da resistência à fratura da estrutura dental, a técnica de amálgama aderido só é relevante frente às cavidades com grande perda tecidual.
10. Eakle WS. Effect of bonded amalgam on the fracture resistance of teeth. J Prosthet Dent 1992; 68:257-60.

11. El-Bradwy WA. Cuspal deflection of maxilary premolars restored with bonded amalgam. J Dent Res 1996; 75:176.

12. El-Mowafy M. Fracture strength and fracture patterns of maxillary premolars with approximal slot cavities. Oper Dent 1993;18:160-6.

13. Hadavi F. Bonding amalgam to dentin [abstract 226] J Dental Res 1993;72:132.

14. Hasegawa T, Retief DH. Shear bond strengths of two commercially available dentin-amalgam bonding systems. J Dent 1996; 24:449-52.

15. Ianzano J. Strength of amalgam restorations bonded with Amalgambond. Am J Dent 1993;6:10-2.

16. Imbery TA, Burgess JO, Batzer RC. Amalgambond, Amalgambond Plus and pinus compared in resistance for amalgams. J Dent Res 1994;73:221.

17. Kim CW, Lim BS. Shear bond strength between dental amalgam and dentin treated with carious bonding agents. J Dent Res 1997;76:68.

18. Lacy AM, Stanine MA. The bonded amalgam restoration. Quintessence Int 1989;20:521-4. 
Minto AMP, Dinelli W, Nonaka T, Thomé LH de C. Estudo comparativo da resistência à fratura de pré-molares superiores íntegros e restaurados com amálgama aderido. Pesqui Odontol Bras 2002;16(2):121-126.

19. Markley MR. Restorations of silver amalgam. J Am Dent Assoc 1951;43:133-46.

20. Miller B, Burgess JO. Shear bond strength of amalgam bonded to dentin [abstract 439] J Dent Res 1997;76:68.

21. Mondelli J. Fracture strength of human teeth with cavity preparations. J Prosthet Dent 1980;43:419-22.

22. Nakabayashi N, Nakamura M, Yasuda N. Hybrid layer as a dentin-bonding mechanism. J Esthet Dent 1991;3:133-8.

23. Ramos JC, Perdigão J. Amalgam bond strength to dentin using current adhesives [abstract 2113] J Dent Res 1997;76:278.

24. Rauvola S. Fracture resistance of bonded versus mechanically retained complex amalgam restorations. J Dent Res 1997;76:67.

25. Sá DN, Gabrielli F. Estudo da infiltração marginal em restaurações com amálgama. Efeito da liga, verniz e brunidura. Rev Fac Farm Odontol Ribeirão Preto 1979;16:53-62.
26. Shimizu A. Amalgam restorations using an adhesive resin cement as a cavity liner. J Osaka Univ Dent Sch 1987; 27:147-55.

27. Staninec M, Holt M. Tensile adhesion and microleakage of resin bonded amalgam restorations. J Prosthet Dent 1988; 59:397-402.

28. Torii Y. Inhibition in vitro of caries around amalgam restorations by bonding amalgam to tooth structure. Oper Dent $1989 ; 14: 142-8$

29. Vale WA. Cavity preparation and further thoughts on high speed. Br Dent J 1959;107:333-46.

30. Varga J, Matsumura H, Matsumura E. Bonding of amalgam filling to tooth cavity with adhesive resin. Dent Mat J 1986;5:158-64.

31. Zidan O, Abdelkereim U. The effect of bonding on the stiffness of teeth restored with amalgam and composite [abstract 2119] J Dent Res 1997;76:278.

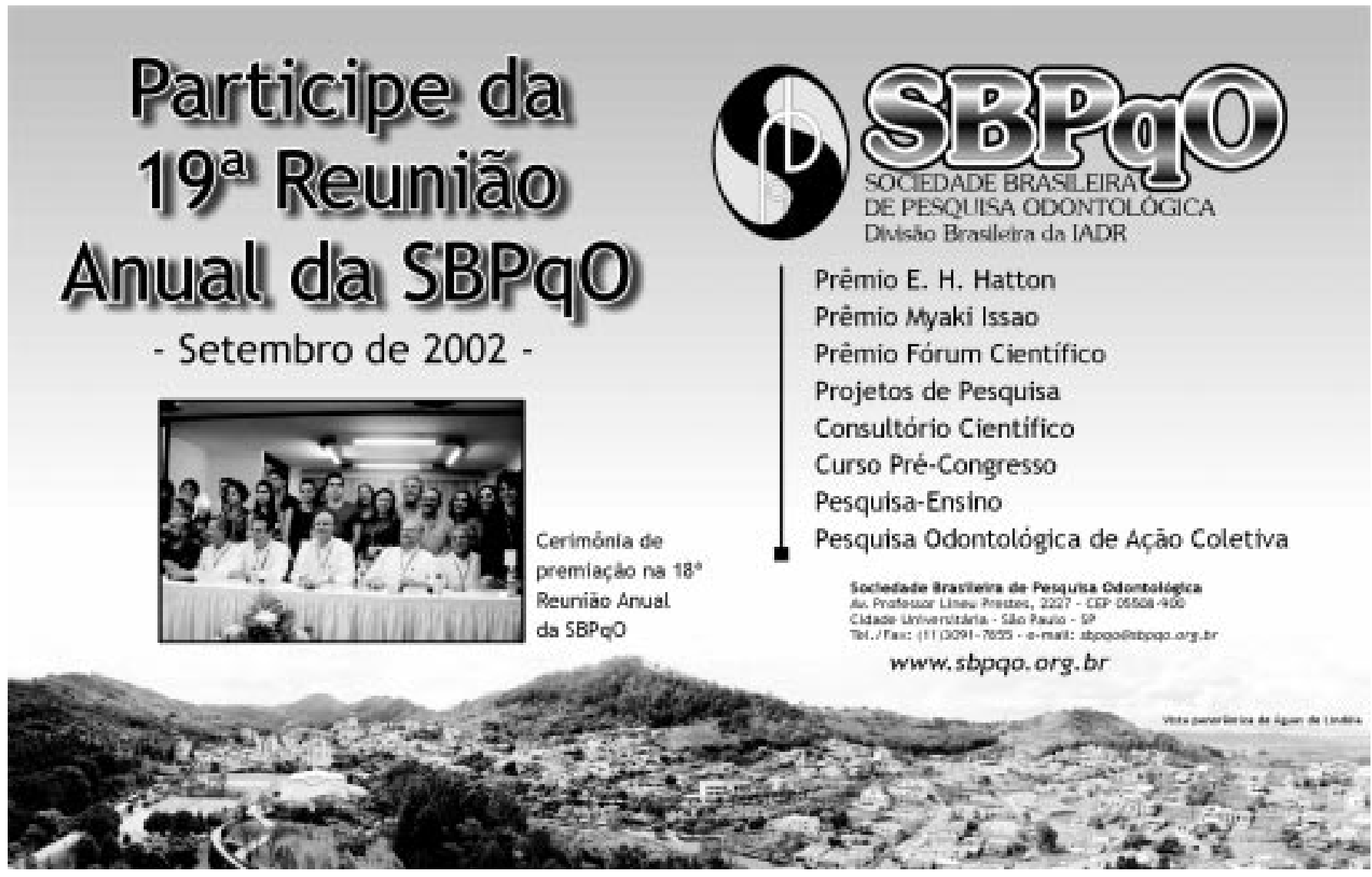

